\title{
Fields in chaos
}

Ian Percival

Nonlinear Physics: From the Pendulum to Turbulence and Chaos. By R.Z. Sagdeev, D.A. Usikov and G.M. Zaslavsky. Harwood: 1988. Pp.675. Hbk \$98, £55; pbk \$39, £21. Six accompanying diskettes $\$ 77, £ 43$.

THE main authors of this book, Sagdeev and Zaslavsky, have made notable contributions to nonlinear physics. A few years ago Zaslavsky moved from Krasnoyarsk to work with Sagdeev at the Space Institute in Moscow, and the book is one result of their collaboration.

It is an ambitious venture, written for the physicist who wants to use nonlinear dynamics, and includes many analytic and numerical methods and results that have been developed in the past few decades. Its particular strength is the recognition of the important role of chaotic dynamics in many fields, particularly plasma physics. The emphasis is strongly on hamiltonian systems and on applications.

The authors begin with the more traditional methods of hamiltonian dynamics, but from a modern point of view. Then follows a section of special methods; these are largely developments of Poincaré's ideas as tools for applications, but include the KAM theorem. The next hundred pages deal with various aspects of chaos, most of it hamiltonian. There is a short chapter reviewing the principles - which derive largely from the work of mathematicians - and then a long one giving a detailed description of some of the complicated and subtle properties of hamiltonian chaos; most of these have been discovered by numerical experiment using computers, but backed by theory. The next chapter, on kinetic theory, concentrates on those aspects of transport which are of import ance in plasma physics.

Part 2, on nonlinear waves, starts with stationary waves, including normal and collision-free shocks, ion-acoustic and magnetosonic waves; the connection made with the theory of particle motion is helpful here. Exactly integrable wave equations are introduced in the context of their applications, and are then treated in more detail later. There follows a description of the hamiltonian formulation, including resonant interaction, resonances and interaction of phonons, and two long chapters on chaos in wave fields and strong turbulence. Strong turbulence is introduced through the Lorenz model, and the distinction between temporal and space-time chaos.

Part 3 gives examples, and is really a collection of bits and pieces that might not have fitted naturally into earlier sections. The first and best is on the motion of particles in wave fields, to which the authors have made important recent contributions and which are illustrated with strikingly beautiful computer-produced colour plates at the end of the book. The second is on billiards, but is too brief to do justice to the subject. The connection with the ray theory of wave optics is considered, but there is no mention of the work of Keller, Maslov, Gutzwiller, Berry and many others on the connection between rays and waves in general, and billiards and cavities in particular.

A later section deals with nonlinear chains, especially with the mathematical equivalence of the equilibrium of incommensurate structures and the dynamics of certain maps. Here, however, there is no

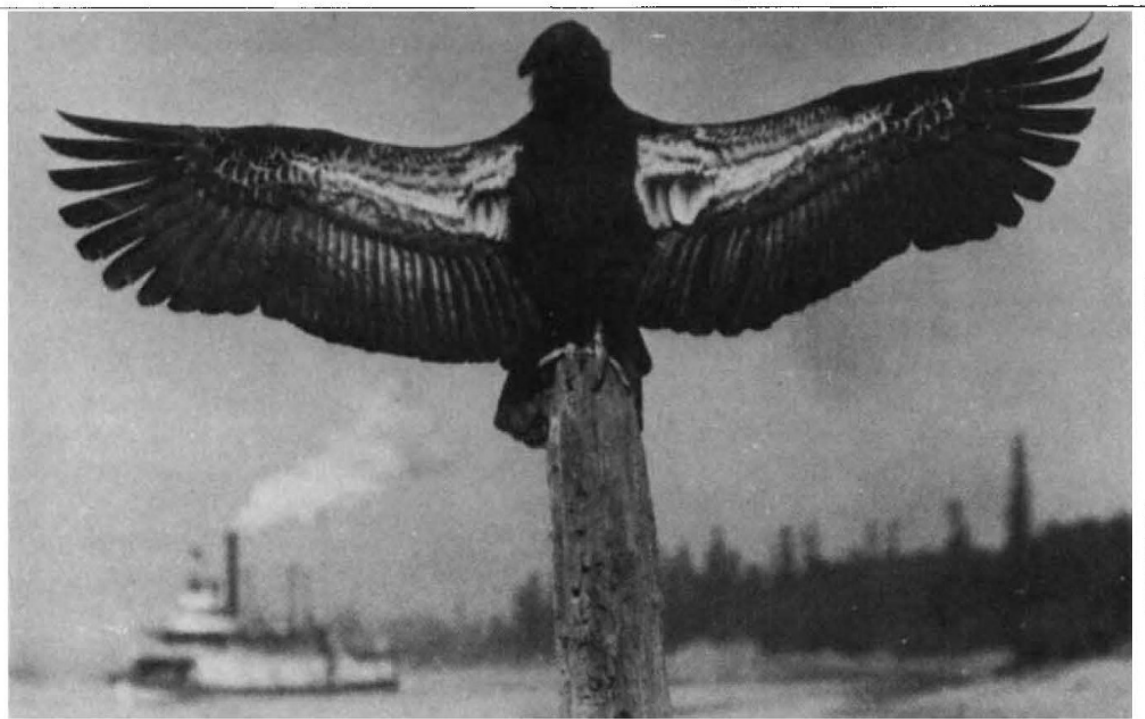

Living totem pole - "Young Condor on Post, Steamboat in Background, Jenning's Lodge, Oregon, 1906", a high-quality photograph on a $5 \times 7$-inch glass plate by W. L. Finley and H. Bohlman. The picture is taken from a chapter on printing old negatives in Alfred A. Blaker's Handbook for Scientific Photography, 2nd edn, just published by Focal Press (an imprint of Butterworth), price $\$ 45, £ 39.95$ description of the Frenkel-Kontorova model, or of Aubry's classic work on its correspondence with the standard map. There are also chapters on applications to nonlinear optics, and to the perturbed Kepler problem (with applications to astronomy and to the excitation of Rydberg atoms). Each provides enough to whet the appetite, but falls far short of being a modern review of the field.

The fourth and final part of the book, by Usikov, is the most unusual. It provides a detailed guide to the programming methods necessary to explore some of the dynamical systems with the aid of an IBMcompatible microcomputer. The detailed listing is not provided, though disks can be obtained at an extra cost.

Part of the problem in writing a book of this kind is that the detailed theory is presented analytically, with some help from geometry, whereas many of the advances are made with the aid of computer experiments, which cannot be put directly into print. So the normal method of presentation is misleading. The final part of the book tries to redress this situation. The 'colour supplement' of orbit simulations is valuable, and will encourage readers to experiment for themselves. But it is not so clear that the computer programs will be so useful, and there is a strong risk that they are becoming outdated - nowadays people use mice!

It is said that Enrico Fermi's use of English was often wrong, but that it was best not to correct it because the errors were minor and had such charm that it was a pity to remove them. Much the same can be said of this book - for example "multiple period birth bifurcations" (p.199) looks more like a term from gynaecology than nonlinear dynamics; but we know what the authors mean. More serious is the use of the words "chaos", "stochasticity" and "turbulence" without the distinctions of modern usage, and the use of "soliton" for interacting solitary waves.

My impression is that the first three parts of the book were written by two busy and productive scientists, who naturally concentrated on the areas to which they have made research contributions. They did not, it seems, have the time to bring all the chapters up to date, nor to provide the polish, revision, indexing, references and cross-references which would have made this a much better volume. For example, although the references provide a guide to some important Soviet literature, they are by no means comprehensive.

Nonetheless this is a valuable contribution to the literature on nonlinear physics. And unlike Zaslavsky's earlier book Chaos in Dynamical Systems, which was terribly overpriced, it is very good value for money, especially in paperback.

Ian Percival is a Professor in the School of Mathematical Sciences, Queen Mary College, Mile End Road, London EI 4NS, UK. 